NASA Technical Memorandum 105675

\title{
Experimental Investigation of the Flowfield of an Oscillating Airfoil
}

J. Panda and K.B.M.Q. Zaman

Lewis Research Center

Cleveland, Ohio

Prepared for the

10th Applied Aerodynamics Conference sponsored by the American Institute of Aeronautics and Astronautics

Palo Alto, California, June 22-24, 1992 


\title{
EXPERIMENTAL INVESTIGATION OF THE FLOWFIELD OF AN OSCILLATING AIRFOIL
}

\author{
By \\ J. Panda* and K.B.M.Q. Zaman ${ }^{* *}$ \\ NASA Lewis Research Center \\ Cleveland, OH 44135
}

\begin{abstract}
The flowfield of an airfoil oscillated periodically over a wide range of reduced frequencies, $0 \leq \mathrm{k} \leq 1.6$, is studied experimentally at chord Reynolds numbers of $R_{c}=22,000$ and 44,000. The NACA0012 airfoil is pitched sinusoidally about one quarter chord between angles of attack $(\alpha)$ of $5^{\circ}$ and $25^{\circ}$. Detailed flow visualization and phase averaged vorticity measurements are carried out for $\mathrm{k}=0.2$ to document the evolution and the shedding of the dynamic stall vortex (DSV). In addition to the DSV, an intense vortex of opposite sign originates from the trailing edge just when the DSV is shed. After being shed into the wake, the two together take the shape of a large "mushroom" while being convected away from the airfoil. The unsteady circulation around the airfoil and, therefore, the time varying component of the lift is estimated in a novel way from the shed vorticity flux and is found to be in good agreement with the lift variation reported by others. The delay in the shedding of the DSV with increasing $\mathrm{k}$, as observed by previous researchers, is documented for the full range of $k$. The DSV, for example, is shed nearly at the maximum $\alpha$ of $25^{\circ}$ at $\mathrm{k}=0.2$, but is shed at the minimum $\alpha$ of $5^{\circ}$ at $\mathrm{k}=$ 0.8 . At low $\mathrm{k}$, the flowfield appears quasi-steady and the bluff body shedding corresponding to the maximum $\alpha\left(25^{\circ}\right)$ dominates the unsteady fluctuations in the wake.
\end{abstract}

\section{Introduction}

The phenomenon of dynamic stall on airfoils and lifting surfaces in unsteady flow environments has been studied experimentally and computationally for many years, both as an important practical problem and a challenging fundamental one as well. The phenomenon appears on helicopter rotor blades, rapidly maneuvering aircraft, fluttering compressor blades, wind turbines and even fish tails and insect wings. It is now well known that the unsteady fluid mechanics of an airfoil pitched sufficiently above the static stall limit, is characterized by the formation of a strong vortex on the suction surface, known as the dynamic stall vortex (abbreviated here as DSV), which is eventually shed into the wake. Presence of the DSV on the airfoil upper surface causes a dramatic increase in the airfoil lift which, however, decreases suddenly when the DSV is shed. Excellent overviews of this dynamic stall phenomenon can be found in the papers by McCroskey ${ }^{1}$ and $\mathrm{Carr}^{2}$.

The flowfield of an oscillating airfoil in a uniform stream is governed by two time scales: one imposed by the pitching motion, $T_{p}=1 /(2 \pi f)$; and the other by the free stream velocity and the airfoil chord (conventionally, half the chord is used), $T_{f s}=c / 2 U_{\infty}$. Here, $f$ is the oscillation frequency, $\mathrm{c}$ the airfoil chord and $\mathrm{U}_{\mathrm{c}}$ the freestream velocity. The reduced frequency, defined as $k=\pi \mathrm{fc} / \mathrm{U}_{\omega}$, thus represents the ratio of the two time scales, $\mathrm{T}_{\mathrm{fs}} / \mathrm{T}_{\mathrm{p}}$. For very low $k$, when $T_{p}>T_{\text {fs }}$ unsteady effects are relatively weak and the flowfield is expected to respond in a quasi-steady manner. At very high $\mathrm{k}$, on the other hand, intuitively one might expect that the outer flow would be unable to respond to the pitching motion and the moving airfoil might appear similar to a bluff body to the flow.

Most of the previous experimental (e.g. Carr et al. ${ }^{3}$; Leishman ${ }^{4}$ ) and computational (e.g., Mehta ${ }^{5}$, Wu et al. ${ }^{6}$ ) studies on the phenomenon are confined to low values of $\mathrm{k}$ $(<0.4)$. Small amplitude pitching motion at very high $\mathrm{k}$ has been studied by Koochesfahani ${ }^{7}$, among others, but the small amplitude $\left( \pm 4^{\circ}\right)$ apparently precluded the possibility of dynamic stall. A low Reynolds number experimental study by Ohmi et al. ${ }^{8}$ covered a higher range of $\mathrm{k}$ but the effect studied was more complex, viz., that due to a combined oscillating and translating motion of the airfoil. The latter study showed that at relatively low $\mathrm{k}(=0.63)$ the flowfield is primarily governed by the translatory motion, but at high $\mathrm{k}(=3.2$ and 6.6$)$ the oscillatory motion controls the flowfield. Gad-el-Hak and $\mathrm{Ho}^{9}$ also covered a wide range of $\mathrm{k}$ and observed the formation of additional vortices due to the motion of the trailing edge. They concluded that the complex flowfield of an oscillating airfoil can be explained primarily from the mutual induction between the DSV and the "trailing edge shedding vortex".

It also becomes apparent from a literature survey that the primary purpose of most previous experiments on dynamic stall was to determine the unsteady forces and

\footnotetext{
* NRC resident research associate Member AIAA

* Senior member AIAA
} 
pitching moments on the airfoil ${ }^{3,4}$. Efforts to quantify the dynamic stall vortex, its evolution and trajectory, and its dependence on the reduced frequency $k$ have been very limited. There have been several computational studies of the flowfield over a pitching airfoil (e. g. Shida et al. ${ }^{10}$, Visbal and Shang $\left.{ }^{11}\right)$. These studies, however, require enormous computational power to obtain grid-independent solution. Moreover, applicability and appropriateness of turbulence models required in the computation of the unsteady, separated flow under consideration are always questionable. Thus further experimental studies on the topic are clearly desirable.

The mechanism for the generation and subsequent accumulation of vorticity leading to the formation of the dynamic stall vortex is discussed by Reynolds and Carr ${ }^{12}$, $\mathrm{Ho}^{13}$ and $\mathrm{Visbal}^{14}$. However, validation of the proposed mechanisms is difficult due to a lack of detailed flowfield measurements. Also, most of the previous flowfield studies concentrated on the DSV while it is located over the airfoil surface, and the structure of the downstream wake remains relatively unknown. In only a few experiments was the vorticity distribution in the wake measured, but these experiments were limited in scope ${ }^{15,16}$. An understanding of the wake structure originating from the dynamic stall process is essential for analysis of more complex flows, e.g., in machinery involving rows of blades ${ }^{17}$. These are the general issues which motivated the present experimental investigation.

The objectives of this investigation are twofold. First, to study the detailed flowfield for a specific k, and secondly, to study the effect of $\mathrm{k}$ on the dynamic stall process. The oscillation amplitude and the mean angle of attack are kept constant. Detailed flowfield measurements are conducted by the phase averaging technique. The distribution of the spanwise component of vorticity is measured by phase averaging of the streamwise and the transverse components of the velocity. The measurements are performed for the actual spatial distribution over the cross-section of the flow, and thus, without invoking the Taylor hypothe$\operatorname{sis}^{18}$. The unsteady vorticity data are analyzed to estimate the unsteady lift force on the airfoil, the concept of which is discussed in the text. Limited quantitative measurements are performed for other values of $\mathrm{k}$ over the range, $0 \leq \mathrm{k}$ s1.6. For all operating conditions, flow visualization is performed by the smoke-wire technique.

\section{Experimental procedure}

The experiments were carried out in a low-speed, $76 \times 51 \mathrm{~cm}$ wind tunnel as shown in figure 1a. The flow enters through a 16:1 contraction section with five screens, passes through the test section and is then exhausted by an axial flow fan. The free-stream turbulence intensity in the test section is less than $0.1 \%$. An NACA0012 airfoil with $10.2 \mathrm{~cm}$ chord and $76 \mathrm{~cm}$ span is mounted horizontally at the center (mid-height) of the test section. The maximum blockage to the flow occuring at the maximum angle of attack of $25^{\circ}$ is $8.5 \%$. The airfoil is supported at the two ends by two $0.635 \mathrm{~cm}$ diameter rods each of which passes through a pair of cylindrical bearings housed in the tunnel walls. The pitching mechanism (figure $1 b$ ) essentially consists of a crank and connecting-rod, together with a flywheel, to oscillate a lever arm. The lever arm (not visible in figure 1b) oscillates an output shaft which in turn is connected to one of the airfoil support rods via a flexible coupling. The oscillation amplitude is adjusted by changing the crank radius and the oscillation frequency is controlled by varying the motor $\mathrm{rpm}$. The entire pitching mechanism is installed in a steel frame which is structurally divorced from the tunnel and is secured to the vibration isolated bed plate of the test cell.

For all data presented here, the airfoil is supported at the quarter chord location and the angle of attack is varied as $\alpha=15^{\circ}+10^{\circ} \sin (2 \pi \mathrm{ft})$. In the following, the suffix " $\mathrm{u}$ " is used to indicate upstroke when $\alpha$ is increasing and " $\mathrm{d}$ " is used to indicate downstroke when $\alpha$ is decreasing. Experiments were conducted at two chord Reynolds numbers, $R_{c}=22,000$ and 44,000 , and for $k$ values of 0 (fixed), .05, .1, .2, .3, .4, .6, .8, 1., 1.2 and 1.6. Experiments at the higher $R_{c}$ were conducted only for $k \leq 0.4$. Detailed measurements and analysis, however, are done for only one case, $\mathrm{k}=0.2(4 \mathrm{~Hz})$ and $R_{c}=44,000$.

The measurements are made using a single or a crossed hot-wire probe mounted on the traversing mechanism shown in figure la. The probe can be traversed in the streamwise $(x)$ direction through a longitudinal slot in the test section floor. For a given $\mathrm{x}$, the probe can be moved up and down in y through an automated computer control. The co-ordinates $x$ and $y$ are referenced to the airfoil trailing edge at $0^{\circ}$ angle of attack, and unless otherwise stated all measurements reported are for the $x-y$ plane at the mid-span location. The probe movement, data acquisition, and analysis were done by a MicroVAX 3300 computer.

The smoke wire technique is used for flow visualization. Essentially the same procedure was used as followed by Panda ${ }^{19}$. A 0.005 inch nichrome wire was placed vertically either upstream of the airfoil or just downstream of the trailing edge. A flash unit was used to illuminate the smoke streaks from the wire which were photographed using a $35 \mathrm{~mm}$ camera. Control on the smoke-wire operation was not fine enough to produce photographs at a desired $\alpha$ during the oscillation. A small rod, marked every .25 inch by white dots, was placed downstream of the trailing edge. Photographs of the marker and the airfoil fixed at different $\alpha$ were taken to calibrate the marker. Usually, a large number of photographs were taken at each flow condition. The position of the airfoil trailing edge relative to the marker determined the approximate angle of attack. The direction of motion 
was inferred from reviewing a large ensemble of photographs.

\section{Experimental results}

Since the flow visualization photographs present the global view of the flowfield, they are discussed first. This is followed by the detailed measurements for the $\mathrm{k}$ $=0.2$ case. The calculation of the unsteady lift from the vorticity flux is discussed next. Limited quantitative measurements on the effect of the reduced frequency $k$ is discussed in the last section.

\subsection{Flow visualization}

\subsubsection{Flowfield for $k=0.2, R_{c}=44,000$}

Figure 2a shows a sequence of photographs at various phases of the oscillation cycle. The flow is from left to right. The marker with white dots is visible at the airfoil trailing edge. Frames $\mathrm{A}$ to $\mathrm{F}$ show phases when the angle of attack $(\alpha)$ is increasing (upstroke) and frames $\mathrm{G}$ to $\mathrm{J}$ show phases when $\alpha$ is decreasing (downstroke). As $\alpha$ increases, a clockwise vortex forms on the airfoil surface (frame D). This is the "dynamic stall vortex" as referred to by previous researchers. With further increase in $\alpha$, the DSV moves towards the trailing edge. When it reaches the trailing edge, a counter-clockwise vortex starts to form near the trailing edge (frame F). This vortex becomes clearer in frame $G$ and can be seen more clearly in figures presented later. The counter-clockwise vortex will be referred to as the trailing edge vortex (TEV) in the following. The TEV grows quickly beneath the DSV (frames F and G) and lifts the latter from the airfoil upper surface. The DSV and the TEV combine to form a structure whose cross section looks like a mushroom. This evolves and increases in size as it moves downstream (Frames H, I and J). In frame I, this vortical structure is about $2 \frac{1 / 2}{2}$ chord lengths from the trailing edge. The transverse extent is already very large and measures about 3 chord lengths.

The presence of the TEV can be seen in some flow visualization photographs and computational results of previous researchers. Gad-el-Hak and $\mathrm{Ho}^{8}$ called it the trailing edge shedding vortex. Flow visualization for single pitch-up motion (pitch rate, $\alpha^{+}=0.1$ ) by Walker and Robinson ${ }^{20}$ showed such a vortex, which they referred to as the "secondary vortex". Shida et al. ${ }^{10}$ showed density contours, obtained computationally, at various phases for the flow around an airfoil oscillating between $0^{\circ}$ and $20^{\circ}$ angles of attack at $\mathrm{k}=0.1$. The results showed an additional region of strong density gradient near the trailing edge at $\alpha=19.3^{\circ}$ when apparently the DSV was being shed. This can be interpreted as a consequence of the TEV. It will be shown later that the formation of the TEV causes a large lift oscillation observed in the lower branch of the hystersis loop in the $C_{\ell}$ versus $\alpha$ curve.

The 'mushroom-like' structure observed in the wake has remained practically unnoticed in previous work. This enormous vortical structure, which occurs periodically at the oscillation frequency, may be quite significant in blade vortex interaction and aerodynamic noise generation from configurations involving rows of blades. After the passage of the DSV and the TEV, frames $\mathrm{J}$ and $\mathrm{A}$ indicate the passage of a few smaller vortices before another DSV begins to form on the upper surface.

Figure $2 \mathrm{~b}$ shows a sequence of smoke-wire photographs for the same flow condition as in figure 2a but with the smoke-wire located near the trailing edge. Frames a, b and c represent phases when $\alpha$ is increasing and frames $d$ and e represent phases when $\alpha$ is decreasing. Frames a,b,c,d,e in figure $2 b$ approximately correspond to frames A,B,E,G and I in figure $2 a$, respectively. When the smoke-wire is kept upstream of the leading edge, the separated flow region on the upper surface and in the near wake remains dark due to the lack of convection of the smoke-containing fluid. However, when the smoke-wire is placed downstream of the trailing edge, strong reverse flow at certain phases carries the smoke upstream, highlighting the reverse flow zones. The photographs of figure $2 b$ clearly show the extent of flow reversal at various phases during the oscillation cycle.

Unlike a steady airfoil (see photographs later), strong reverse flows can be observed even at the minimum $\alpha=5^{\circ}$ for the oscillatory case (figure $2 b$ ). A computational study of the flowfield for an airfoil undergoing a single pitch-up motion by Visbal ${ }^{14}$ also showed the presence of a strong reverse flow in a thin layer adjacent to the upper surface during the formation of the dynamic stall vortex. Once the separated flow reattaches and the DSV starts to form (frames B and $b$, figures $2 a$ and $2 b$ ), no reverse flow is visible. Presumably, the flow is attached to the airfoil upper surface downstream of the DSV and thus the smoke is not entrained upstream. However, it should be obvious that the DSV is accompanied by regions of reverse flow adjacent to the airfoil surface.

\subsubsection{Effect of the reduced frequency $\mathrm{k}$}

Flowfield for $k=0$, different $\alpha$ : Figure 3 shows visualization photographs of the fixed airfoil for three indicated values of $\alpha$. The pictures in the right column are taken with the smoke-wire placed upstream of the leading edge and those on the left column are taken with the smokewire placed downstream of the trailing edge. The NACA0012 airfoil undergoes complete stall at about $\alpha=14^{\circ}$. The photographs for $\alpha=15^{\circ}$ and $25^{\circ}$ show fully stalled flow. Due to the low operating Reynolds number, it is 
apparent that a "laminar separation" has taken place even when the airfoil is at $5^{\circ}$ angle of attack. It is interesting to note that with the smoke wire placed at the trailing edge, the separated region remains dark for $\alpha=5^{\circ}$. This indicates weak reverse flow under laminar separation. Experiments on laminar separation also showed weak reverse flows in such a situation ${ }^{21}$. Referring back to figure $2 b$, note the difference with the oscillating airfoil case where at the same $\alpha$ there is a strong reverse flow.

At larger angles of attack the airfoil wake is characterized by the Karman vortex street (alternatively referred to as the bluff-body shedding) which contains alternate clockwise and counter-clockwise vortices. The corresponding Strouhal number $\mathrm{St}_{\mathrm{s}}=\mathrm{f}_{\mathrm{s}} \mathrm{cSin} \alpha / \mathrm{U}$. was measured ( $f_{s}$ being the shedding frequency), to be 0.18 , via spectral analysis of the u-signal. This is consistent with pervious observations ${ }^{22}$.

Flowfield at different $k$ : Figure 4 shows the flowfields for $\mathrm{R}_{\mathrm{c}}=22,000$ at different reduced frequencies, $\mathrm{k}$. These photographs represent the approximate phases when the dynamic stall vortex is lifted up and the trailing edge vortex has clearly formed. At the lowest $\mathrm{k}(=0.05)$ the DSV and the TEV are not clearly visible and the flowfield appears to be in a quasi-steady state. As $\mathrm{k}$ is increased above 0.1 , the unsteady effects become prominent. The initiation of the "mushroom" structure is clear for the higher $\mathrm{k}$ cases.

An interesting effect of varying $\mathrm{k}$ is the resulting phase delay in the shedding of the DSV. At $k=0.2$, the shedding of the DSV takes place when the airfoil is nearly at the end of the upstroke, at $\alpha=24.5^{\circ}$. However, as $\mathrm{k}$ is increased, the shedding is delayed to the downstroke when $\alpha$ is decreasing. At $\mathrm{k}=0.8$, the DSV is shed when the airfoil is at the minimum angle of attack of $5^{\circ}$. A further increase in $\mathrm{k}$ brings the shedding of the DSV back to the upstroke part of the cycle. Similar observations were made by McAlister et al. ${ }^{23}$, Kim and Seung ${ }^{24}$ and Leishman ${ }^{4}$ in the limited $\mathrm{k}$ range of 0.05 to 0.25 . With increasing $\mathrm{k}$, a delay in the formation of the DSV was observed in the last experiment. In the experiment by Kim and Seung, a similar delay was observed in the formation of the separation region near the trailing edge. McAlister et al.'s data are similar to the present data and are further discussed in the following in connection with figure 5 .

From photographs similar to those in figure $2 \mathrm{a}$, for $\mathrm{k}=0.2$, it is observed that the DSV starts to form at about $\alpha=8^{\circ}$ during the upstroke. In this case, the time elapsed until the airfoil reaches $\alpha=25^{\circ}$, is enough for the DSV to move to the trailing edge and shed into the wake. However, when $\mathrm{k}$ is increased, the time needed by the DSV to form, grow and move to the trailing edge becomes larger compared to the time taken by the airfoil to complete the upstroke. This apparently causes the observed phase delay. At $\mathrm{k}=0.8$, the time needed by the DSV from formation (at the beginning of upstroke) to shedding equals the oscillation period and thus, the shedding occurs at the end of the downstroke. A further increase in $\mathrm{k}$, to say 1.6 , causes two DSVs: one that has started to grow at the leading edge and one left from the previous cycle near the trailing edge, to reside on the airfoil suction surface simultaneously.

Figure 5 shows a plot of the angles of attack and the phase angle $(\omega t)$ at which the center of the DSV is approximately above the trailing edge before it sheds into the wake at various $\mathrm{k}$. The values of $\alpha$ and $\omega \mathrm{t}$ are related by $\alpha=15^{\circ}+10^{\circ} \sin (\omega \mathrm{t})$. Each data point is an estimate from several photographs similar to those shown in figure 4. The phase data are cross checked with $\langle U\rangle$ contours, measured near the trailing edge as discussed later. The solid line in figure 5 represents data taken from McAlister et al. ${ }^{23}$ for the same oscillation condition but at a much higher Reynolds number $\left(2.5 \times 10^{6}\right)$. The latter data represent the instants when the suction peak near the leading edge collapses. The good agreement of these data with the present result, within the k-range covered, indicates that the phase delay phenomenon for comparable airfoils may be essentially independent of the Reynolds number. However, McAlister et al. also showed that leading edge modification such as by a boundary layer trip can significantly alter the phase delay characteristics.

The delay in shedding of the DSV may have a significant effect on the lift and pitching moments experienced by the airfoil at various $\mathrm{k}$. It is known that the lift augmentation takes place with the formation of the DSV and the sudden drop of lift occurs when it leaves the airfoil surface. For low k, maximum lift as well as the sudden drop in lift occurs around the maximum angle of attack, $\alpha=25^{\circ}$. At higher $\mathrm{k}$, the shape of the hystersis loop in the $C_{\ell}$ versus $\alpha$ curve is likely to change. For example, at $\mathrm{k}=0.8$, the maximum lift may occur around $\alpha=5^{\circ}$. For still higher $\mathrm{k}$, when two or more vortices exist on the upper surface simultaneously, complex hystersis loops may be expected in the $C_{\ell}$ versus $\alpha$ curves.

Figure 6 shows pictures similar to those in figure 4 but for different phases. The full grown DSVs (left column), and the "mushroom vortices" (right column), are shown for a few values of $\mathrm{k}$. Again, neither the DSV nor the mushroom vortex are clear for $\mathrm{k}=0.05$. As $\mathrm{k}$ is increased, the DSV becomes stronger and the vortical motion in the core becomes intense. An expected trend with increasing $\mathrm{k}$ is the decrease in the spacing (wavelength) of the mushroom vortices in the wake. For $\mathrm{k}=$ 0.2 , only one mushroom vortex is seen in each frame covering a streamwise distance of about 3 chords, while for $\mathrm{k}=1.6$, spacing between two mushroom vortices decreases to 1.5 to 2 chords. 
At $\mathrm{k}=0.2$, for which detailed flow visualization was shown earlier, the sequence of vortex formation is such that the DSV and the TEV together leave the trailing edge, and this is followed by the shedding of smaller vortices. As $\mathrm{k}$ is increased to 0.4 or higher, the scenario becomes somewhat different. A small clockwise vortex rolls up on the upper surface and is shed in the wake before the dynamic stall vortex; all other smaller vortices are suppressed. McAlister and $\mathrm{Carr}^{25}$ observed this smaller vortex preceding the DSV in their flow visualization experiment and called it the "shear layer vortex". The present visualization pictures also appear to show that this vortex is accompanied by another counterclockwise vortex prior to the shedding of the DSV.

Note that at even higher $\mathrm{k}$, there is a significant change in the "mushroom" structure. From figure 6 it can be seen that while the structure appears upright at $\mathrm{k}=$ 0.6 , it is tilted for $\mathbf{k}=1.2$, and appears upside down at $\mathrm{k}=1.6$. The vortex structures for $\mathrm{k}=0.6$ and 1.6 are sketched in figure 7. At the lower $\mathrm{k}$, the DSV is shed before the TEV and both of them move upwards in positive $y$. On the other hand, at $\mathrm{k}=1.6$, the TEV is shed earlier than the DSV and the resulting "inverted mushroom" remains in the same horizontal plane containing the pitch axis. A somewhat intermediate structure is seen for $\mathrm{k}=1.2$.

A question may be asked if there exists an optimum range of $\mathrm{k}$ for which the vortices due to dynamic stall are the strongest and the largest. The visualization photographs shown in the preceding figures indicate that, within the distance covered in these photographs, the DSV and the TEV grow to the largest size for values of $k$ in the range of $0.2-0.4$ (see e.g., frame I, figure 2a). As $\mathrm{k}$ is increased further, the DSV as well as the "mushroom vortex" reduce in size. However, the vortical structures become more complex at larger $\mathrm{k}$.

\subsection{Flowfield measurements for $k=0.2, R_{c}=44,000$ 3.2.1 Phase averaged vorticity measurements}

The axial and transverse velocity components, ensemble averaged over many oscillation cycles, are measured in the wake as well as on the suction side of the airfoil, avoiding the region covered by the pitching motion. The periodic phase reference, once in every oscillation cycle, is obtained by an optical pick-up mounted on the pitching mechanism (figure $1 \mathrm{~b}$ ). At first, the signals from the crossed hot-wire probe and the optical pick-up are stored in a digital computer. The data are acquired at several $\mathrm{x}$-stations. At each $\mathrm{x}$-station the automated traverse is used to collect data at several transverse locations (y). These data are post-processed to obtain the phaseaveraged distributions of $\langle\mathrm{U}\rangle$ and $\langle\mathrm{V}\rangle$. Typically the averaging is done over 80 cycles or more. Finally, the spanwise vorticity component is calculated as,

$$
\left\langle\omega_{z}\right\rangle(x, y, \tau)=\frac{\partial<V\rangle}{\partial x}-\frac{\partial\langle U\rangle}{\partial y}
$$

The vorticity values are non-dimensionalized by $U_{\omega} / c$.

Figure 8 shows examples of the phase averaged $\langle\mathrm{U}\rangle$ profiles at three axial locations for five oscillation phases. For a given $\mathrm{x}$, the instantaneous "wake profiles" vary dramatically with oscillation phase. At $x / c=-0.3$, the amplitudes are likely to be contaminated by hot-wire error due to reverse flow close to the airfoil surface ( $y / c$ $<0.15$ ); the errors are discussed further in the following. The highest $\langle U\rangle$ variation at $x / c=0.6$ is observed around the maximum $\alpha$ of $25^{\circ}$ when the DSV and the TEV pass through the measurement station. The profile showing maximum variation of $\langle\mathrm{U}\rangle$ shifts, due to the convection time taken by the mushroom structure, to a later phase at the downstream station of $x / c=1.5$. Velocity profiles similar to those shown in figure 8 , as well as the corresponding $\langle\mathrm{V}\rangle$-profiles, were obtained for 50 phase points covering a complete cycle and $32 \mathrm{x}$-stations.

Equi-velocity contours of phase averaged $\langle\mathrm{U}\rangle$ (left column) and $<\mathrm{V}>$ (right column) are shown in figure 9; the instantaneous values of $\alpha$ are indicated. The measurement domain is divided into two parts. The first region lies on the airfoil upper side: $-0.75 \leq x / c \leq 0.1$ and $0.1 \leq \mathrm{y} / \mathrm{c} \leq 1.0$ and is divided into $13(\mathrm{y}) \times 12(\mathrm{x})$ measuring stations; the second region covers the wake from near the trailing edge, $.06 \leq x / c \leq 2.06$ and $-.75 \leq y / c \leq 1.0$, and is divided into 20 (y) X 17 (x) stations. Since results from these two sets are patched together in each frame of figure 9, there is some mismatch at the interface. The mismatch is caused partly by measurement error and mostly by a slight phase drift between the two sets of measurements. As will be clear from the vorticity plots (shown shortly), the top row of figure 9 corresponds to an instant when large vortices are absent in the measurement domain. The middle row corresponds to a phase when the DSV is moving towards the trailing edge on the airfoil upper surface. The DSV is characterized by strong axial and transverse velocities around its core. The bottom row shows the velocity field when the "mushroom vortex" structure occupies the wake. These data clearly show the enormous changes in the unsteady flowfield that takes place periodically under the action of the vortical structures. The peak-to-peak variations in $\langle U\rangle$ and $\langle\mathrm{V}\rangle$ can be observed to be comparable in these data.

As indicated before, a crossed hot-wire probe was used to measure the velocity components, following similar procedure as used by Westphal and Mehta ${ }^{26}$. There are some errors in these measurements, especially due to hot-wire rectification during instants of large flow angularity and flow reversal. Even without rectification the errors are significant when the instantaneous velocity vector lies outside $\pm 30^{\circ}$ of the direction bisecting the 
probe wire angle. There is no easy way to assess the errors accurately and the exact extent of the errors has remained unknown. The flow visualization photographs have shown that flow reversal occurs close to the airfoil upper surface when the DSV and the other vortices pass by. The measurement domain, however, excludes the regions near to the airfoil surface. Nevertheless, some flow reversal and large flow angularity are likely to have contaminated the data especially in the middle of the wake just downstream of the trailing edge. Contours of the flow angle $\left.\left(\tan ^{-1}<\mathrm{V}\right\rangle \mid\langle\mathrm{U}\rangle\right)$ were constructed from the measured $\langle U\rangle$ and $\langle V\rangle$ data (not shown). Downstream of $x / c=0.25$, the phase averaged flow angle was found to be confined in the range of $-26.5^{\circ}$ to $+31.5^{\circ}$, for all measurement points at all phases. Inspection of the contours (which look very similar to the $\langle\mathrm{V}\rangle$-contours) also indicates that the data are free from rectification. On this basis, it is inferred that the errors due to large flow angularity are not significant downstream of one quarter chord from the trailing edge, as well as for $y / c>0.15$ for the upstream measurement region above the airfoil. Good comparison of the measured vorticity field (discussed shortly) with the flow visualization photographs supports this conclusion.

\subsubsection{Vorticity evolution}

The evolution of the spanwise component of vorticity at various phases is shown in figure 10 . For $\mathrm{k}$ $=0.2$, the distance through which the flow structures are convected downstream in one period of oscillation is much longer than that covered by the measurement domain. Hence, each frame shows the wake structure for a small fraction of one complete cycle. The data acquisition rate was chosen such that the phase-averaged distributions are computed for 50 time instances per cycle. However, only 16 time instances, clearly capturing the passage of the vortices, are shown in figure 10. Note that the frames are not at equal time intervals.

During the initial part of the cycle, as $\alpha$ is increased, the measurement region is relatively quiet and large vortices are absent. The wake region is marked by clockwise vorticity above the trailing edge, and counterclockwise vorticity below it. As the angle of attack is increased above $8^{\circ}$, a small vortical region nucleates near the airfoil quarter chord and grows rapidly with accumulation of positive vorticity. Note that the equi-vorticity contours are drawn up to a maximum level of 12. Peak levels exceeding this value have been marked in some of the regions. The nucleation of positive vorticity over the airfoil is accompanied by an interesting change in the wake vorticity distribution. The negative vorticity in the lower part of the wake remains as is, but the positive vorticity in the upper part of the wake depletes dramatically (see, e.g., data for $\alpha=20.7 \mathrm{u}$, figure 10). The positive vorticity, generated on the airfoil suction surface, which otherwise would have shed into the wake, starts to accumulate leading to the formation of the dynamic stall vortex. A similar process is described by Visbal ${ }^{14}$ from his computational results.

Increasing $\alpha$ above about $18^{\circ}$ causes a large portion of the accumulated vorticity to move towards the trailing edge. This represents the dynamic stall vortex and is marked as V1 in figure 10. The shedding of the DSV in the wake (around the phase marked 24.9d), is accompanied by the appearance of the counter-clockwise trailing edge vortex. Initially the TEV, marked V2, is small and lies underneath the DSV. However, as the two evolve, the TEV moves upward. The combination of the DSV and the TEV gives the appearance of a mushroom in the flow visualization photographs. During the downstroke, positive vorticity is shed continuously in the wake which rolls-up to form vortices V3 and V5. It is interesting to note that each of the clockwise vortices is followed up by a counter-clockwise vortex (V4, V6). Therefore, the wake contains a series of clockwise and counter-clockwise vortices after the passage of the DSV and the TEV. The succession of a counter-clockwise vortex following the passage of a clockwise vortex is, perhaps, expected as the net vorticity shed into the wake over a complete oscillation cycle should be zero. The measured vortical structures, their streamwise and transverse extents, agree quite well with the flow visualization photographs of figures $2 a$ and $2 b$.

\subsubsection{2-dimensionality of the flowfield}

In order to check the spanwise uniformity of the flowfield, phase averaged velocity measurements were conducted at various spanwise $(\mathrm{z})$ stations for constant $\mathrm{x}$ and $y$. Figure 11 shows a set of $\langle U\rangle$ traces at different $z / c$, for $x / c=1.0$ and $y / c=0$. More than one period of oscillation is shown in every trace. Passage of the dynamic stall vortex is marked by large positive peaks in the $<U>$ traces. The velocity traces are quite similar and it is clear that the dynamic stall vortex arrives nearly at the same instant at all spanwise stations. Thus, the flowfield under study may be considered as reasonably 2 -dimensional.

\subsection{Estimation of unsteady lift from the vorticity data}

An effort was made to estimate the time dependent component of the lift from the measured vorticity data. The variation of the unsteady component of lift over an oscillation period may be compared with the hystersis loop in $\mathrm{C}_{\ell}$ variation with $\alpha$ measured by others ${ }^{27}$. The underlying idea and the results are discussed in the following.

Let us consider the control volume $\mathrm{ABCD}$ around the airfoil as shown in figure 12. It is reasonable to assume that the flows at the boundaries $\mathrm{DA}, \mathrm{AB}$ and $\mathrm{BC}$ are irrotational and all vortical fluid passes through 
boundary $\mathrm{CD}$. If the airfoil is held fixed at a given $\alpha$, then the circulation around the path $\mathrm{ABCD}, \Gamma_{0}$, is a constant and the lift $\mathrm{L}_{0}$ is obtained via the Kutta-Joukowskii theorem, $\mathrm{L}_{0}=\rho \mathrm{U}_{\Gamma_{0}}$. For the steady flow, $\Gamma_{0}$ is equal in magnitude but opposite in sign to the total circulation shed in the starting vortex system. After the passage of the starting vortex system, the circulation around the airfoil remains constant and the net vorticity flux passing through the boundary $\mathrm{CD}$ at all times should be zero $^{28}$. In other words, at any time after the passage of the starting vortex system, the amount of positive and negative vorticity passing through the boundary $C D$ should be equal and cancel each other so that $\int_{\mathrm{CD}} \omega_{\mathrm{z}} \mathrm{dy}$ $=0$.

Let us now consider that the airfoil oscillates periodically with a time period $T_{p}$. The circulation, $\Gamma(t)$, around $A B C D$ is now a function of time, varying periodically with period $T_{p}$. At any instant the lift $L(t)$ may be approximated by the Kutta-Joukowskii theorem, $\mathrm{L}(\mathrm{t})=$ $\rho U_{\infty} \Gamma(t)$ (see farther discussion at the end of this section). The vorticity flux through $\mathrm{CD}$ at any instant is now non-zero, and the net vorticity passing over a time $\Delta t$ should be equal and opposite to the change in the circulation $\Delta \Gamma$. Note that the vorticity flux through CD at any instant corresponds to the change in the circulation $\triangle \Gamma$ around $A B C D$ at the same instant. Therefore, the vorticity flux through $C D$ represents the variation in the unsteady circulation around $A B C D$ without any time lag. If we assume that in time $\Delta t$ the vorticity crossing the boundary $\mathrm{CD}$ is contained within the region CDD'C' (figure 12) then,

$$
\Delta \Gamma=\Gamma(t+\Delta t)-\Gamma(t)=\int \omega_{z} d x d y
$$

Where, $\int \omega_{z} \mathrm{~d} x \mathrm{dy}$ represents circulation around CDD'C'. Invoking the Taylor hypothesis and using a suitable average convection velocity, $\mathrm{U}_{\text {conv }}$, one can write,

$$
\Delta \Gamma=-U_{\text {conv }} \Delta t \int_{C D} \omega_{z} d y
$$

Where, the vorticity flux $\int_{\mathrm{CD}} \omega_{\mathrm{z}} \mathrm{dy}$ is a function of time. Use of a constant convection velocity in the Taylor hypothesis should represent a good approximation of the dynamics of the large scale vortical structures ${ }^{18}$, and therefore, the above equation should be a good estimate of $\Delta \Gamma$. Letting $\xi(t)=\int_{\mathrm{CD}} \omega_{\mathrm{z}} \mathrm{dy}$ it follows in the limit that

$$
\begin{gathered}
\frac{d I^{\prime}}{d t}=-U_{\text {conv }} \xi(t) \\
\Gamma(t)=-U_{\text {conv } \int_{t_{0}}^{t} \xi(t) d t+\Gamma\left(t_{0}\right)}
\end{gathered}
$$

Thus if the integration of the vorticity flux is started at time $t_{0}$, the unsteady component of circulation $\Gamma(t)-\Gamma\left(t_{0}\right)$ will be given by the integral in the above equation for any time t. Obviously, the integral over a complete period $\mathrm{T}_{\mathrm{p}}$ should be zero as the magnitude of circulation should come back to the same value as that at the start of the period, i.e., $\Gamma\left(t_{0}+T_{p}\right)=\Gamma\left(t_{0}\right) . \Gamma\left(t_{0}\right)$ represents circulation at time $t_{0}$ and remains unknown. However, the integration of the vorticity flux, carried through different intervals provides the unsteady component of circulation over a period. This in turn provides the unsteady component of the lift $\mathrm{L}_{\mathrm{u}}(\mathrm{t})$ as a function of time (and therefore as a function of $\alpha$ ) over a period.

In the present case, the vorticity measurements are not so reliable near the trailing edge due to hot-wire errors (see section 3.2.1). Thus the integration of the vorticity flux is carried out at a downstream location $\mathrm{x}$, over the boundary EF (figure 12), where measurements are relatively free from such errors. This, however, introduced a phase lag for the calculated variation of the circulation which can be estimated by applying the Taylor hypothesis once again. If $8 \mathrm{t}=8 \mathrm{x} / \mathrm{U}_{\text {conv }}$ represents the time taken by the flow to reach EF from CD then,

$$
\Gamma(t)=-U_{\text {conv }} \int_{t_{0}-\delta t}^{t-\delta t} \xi_{1}(t) d t+\Gamma\left(t_{0}\right)
$$

Where, $\xi_{1}(t)$ is the vorticity flux through EF. The unsteady lift component at any instant of time is given as,

$$
\mathrm{L}_{\mathrm{u}}(t)=\rho U_{\infty}\left(\Gamma(t)-\Gamma\left(t_{0}\right)\right)=-\rho U_{\infty} U_{\text {conv }} \int_{t_{0}-\delta t}^{t-\delta t} \xi_{1}(t) d t
$$

The unsteady component of the lift coefficient, normalized by $0.5 \rho \mathrm{U}_{\infty}{ }^{2} \mathrm{c}$, is plotted as a function of $\alpha$ in figure $13 \mathrm{a}$. In the present calculation, $\mathrm{U}_{\text {conv }}=0.6 \mathrm{U}_{\infty}$ is assumed based on the average convection velocity of the "mushroom vortex". The calculation is started when $\alpha=$ $5^{\circ}$ at the beginning of the upstroke. The axial station where the integration is performed is at $\mathrm{x} / \mathrm{c}=0.3$, where the hot-wire measurement errors should no longer be a serious problem. It is worth mentioning here that varying the calculation station within the range $0.2 \leq x / c \leq 1.5$ produced an essentially similar $C_{\ell}$ variation as shown in figure $13 a$.

The loop in the calculated $\mathrm{C}_{\ell}$ vs. $\alpha$ in figure $13 \mathrm{a}$ does not close completely over one complete period. The amount by which the loop fails to close, reflects the cumulative error over one complete period (50 phase points) in determining the vorticity distribution at $\mathrm{x} / \mathrm{c}=$ 0.3. In spite of this error, the overall features of the calculated $\mathrm{C}_{\ell}$ variation are remarkably similar to the measurements of McAlister et al. ${ }^{27}$ reproduced in figure $13 \mathrm{~b}$. Note that while figure 13a shows the unsteady component 
only, the data of figure $13 \mathrm{~b}$ includes the steady component.

As $\alpha$ is increased, the dynamic stall vortex forms and increases in strength. The slope of $\mathrm{C}_{\ell}$ versus $\alpha$ curve increases as the steady stall limit is exceeded. A comparison with the flow visualization photographs of figure 2 and vorticity evolution of figure 10 shows that the sudden drop in lift occurs when the DSV approaches the trailing edge and is lifted up from the airfoil surface. The rebounding of the lift near the highest angle of attack during the downstroke is due to the shedding of the trailing edge vortex that effectively increases the circulation $\Gamma(t)$ once more. The present data are characterized by undulations in the lower branch of the hystersis loop. The passage of the combined DSV and the TEV is followed by a few other smaller positive and negative vortices causing the observed undulations.

In spite of the overall similarity, clearly differences exist between the data of figures 13a and $13 b$. Some of the differences may be attributed to different experimental conditions between the present experiment $\left(R_{c}\right.$ $\left.=4.4 \times 10^{4}, \mathrm{k}=0.2\right)$ and that of McAlister et al. $\left(\mathrm{R}_{\mathrm{c}}=\right.$ $\left.5 \times 10^{5}, \mathrm{k}=0.15\right)$. It should be emphasized here that large differences can occur in the hysteresis loop especially for different $\mathrm{k}$, as clearly shown by McAlister et $\mathrm{al}^{27}$. and by the wealth of data obtained in earlier experiments at NASA Ames (see, e.g., Carr et $\mathrm{al}^{3}$.). Other factors, e.g., Reynolds number, aspect ratio of the airfoil and leading edge modification can also produce some changes in the hysteresis loop. The data in figure $13 \mathrm{~b}$ were chosen from the cited reference such that the experimental conditions were the closest to those in the present case. However, note that both $\mathrm{k}$ and $\mathrm{R}_{\mathrm{c}}$ are quite different, and therefore, the differences between the two sets of data may not be surprising. There is also another important difference between the present calculation and the force measurement data. The force exerted by the fluid on an oscillating airfoil can be viewed as a result of two contributions. The first one is due to the circulation around the airfoil and the second one is an inertia force associated with the mass of the fluid displaced by the airfoil $\left(\mathrm{Wu}^{28}\right)$. The present calculation scheme approximates the unsteady part of the former, but the latter, which can be shown to be small for low $\mathrm{k}\left(\mathrm{Visbal}^{14}\right)$, is not accounted for.

A conspicuous difference between the present result (figure 13a) and that in figure $13 \mathrm{~b}$ is the undulations in the lower branch of the $\mathrm{C}_{\ell}$ curve. As mentioned before, the rebounding of the lift in the present data is followed by the undulations due to the alternate positive and negative vortices succeeding the TEV. Such undulations have been observed in other experiments (e.g., Leishman ${ }^{4}$ ) and also in certain computational studies. Figure $13 \mathrm{c}$ shows a set of computational result obtained by a two-dimensional Navier Stokes code (L. N. Sankar, private communication). For details of the computational procedure the reader may refer to Wu, Huff and Sankar ${ }^{30}$. Even though these data are for a much different $R_{c}$ and a different $\mathrm{k}$, and the use of turbulence models in unsteady flow calculations is subject to question, the overall features and the undulations in the lower branch are very similar to the present measurements.

An advantage of the present procedure for determining the unsteady component of the lift is that it does not require pressure distribution or force measurements. The data is obtained solely from wake surveys. The pressure measurements may suffer from averaging effect due to inadequate distribution of the pressure taps as well as possible uncertainties due to inadequate dynamic response of the transducers.

Apart from the hot-wire errors, that apparently lead to an unclosed hystersis loop, perhaps the main questionable aspect in the present calculation is the applicability of the Kutta-Joukowskii theorem. If the DSV is lifted up from the airfoil surface but still remains in the control volume $A B C D$ in figure 12 , the actual lift on the airfoil may drop; but, the circulation around the circuit $A B C D$ would remain the same. Thus, the lift variation may not be truly represented by the circulation around $A B C D$. Note also that the latter is only approximated in the present calculation. Another source of error, related to the above problem, arises from the application of the Taylor hypothesis and the accurate estimation of the phase lag between the calculated force and the actual force experienced by the airfoil.

\subsection{Wake velocity spectra:}

The effect of pitching at various oscillation frequencies on the wake velocity spectra, measured one chord downstream and 0.6 chord above the pitch axis, is shown in figure 14 . When the airfoil is not oscillating $(\mathrm{k}$ $=0$ ) and it is at the maximum angle of attack of $25^{\circ}$, a strong peak at a Strouhal number $\mathrm{St}_{\mathrm{s}}=\mathrm{f}_{\mathrm{s}} \mathrm{cSin} \alpha / \mathrm{U}_{\mathrm{s}}=$ 0.18 (where the shedding frequency, $\mathrm{f}_{\mathrm{s}}=26 \mathrm{~Hz}$ ) is observed in the velocity spectrum. This peak corresponds to the bluff body shedding as discussed in connection with figure 3. When the airfoil is oscillated at low $\mathrm{k}$, the $\mathrm{u}$ 'spectrum contains two peaks: one at the oscillation frequency and a second one at the bluff body shedding frequency corresponding to the maximum $\alpha$. Flow visualization photographs for low $\mathrm{k}$, similar to those in figure 4 , do indicate the existence of the Karman vortex street and confirm the quasi-steady behavior of the flowfield.

Since the bluff body shedding frequency is a function of the length scale $\mathrm{cSin} \alpha$, it may be expected that over a period when $\alpha$ varies the vortex shedding will take place over a wide range of frequencies. However, the velocity spectra show a single peak at $\mathrm{fcSin} \alpha / \mathrm{U}_{\infty}=$ 0.18 , where $\alpha$ corresponds to the maximum angle of 
attack. Since the shed vortices are the strongest and the airfoil dwells longer at the maximum $\alpha$, the flowfield is dominated by vortices shed at this $\alpha$. As the reduced frequency is increased the amplitude of the spectral peaks corresponding to the bluff body shedding decreases. Above $\mathrm{k}=0.2$ the bluff body shedding is completely suppressed and multiple harmonics of the pitching frequency appear in the velocity spectra. Again, this trend in the spectral data is in reasonable agreement with the flow visualization pictures discussed earlier. Since, a further increase in the oscillation frequency does not change the overall characteristics of the velocity spectra, spectra for $\mathrm{k}>0.2$ are not shown.

\subsection{The unsteady wake at fixed $x$ for different $k$ :}

Since the actual vorticity measurement, via the spatial data acquisition technique, involves extensive time and effort, such measurements were performed for only a single value of $\mathbf{k}(=0.2)$. In order to further study the effect of $k$, phase averaged measurements were performed for a fixed $\mathrm{x}$-station $(\mathrm{x} / \mathrm{c}=0.12)$ at different $\mathrm{k}$. The $\mathrm{x}$ station close to the trailing edge was chosen to clearly capture the phase delay in the shedding of the DSV with increasing $\mathrm{k}$, even though there is some hot wire error at this station in the middle of the wake (section 3.2.1). Figure 15 shows the contours of $\langle U\rangle$ for six different k. The abscissa in these plots represents the phase position $(\tau)$ normalized by the period of oscillation (Tp).

Let us, at first, consider the data for $\mathrm{k}=0.2$ for which detailed measurements and flow visualizations have been discussed. The appearance of the dynamic stall vortex is characterized by a region of large $\langle U\rangle$ around $\tau / \mathrm{T} p=0.35$ which correspond approximately to the angle of attack of $23^{\circ}$. This, in time, is followed by a thinner wake structure until the minimum $\alpha$ of $5^{\circ}$ is reached at $\tau / \mathrm{Tp}=0.9$. This trend remains qualitatively the same for the other $\mathrm{k}$ values. However, as $\mathrm{k}$ is increased, the appearance of the DSV at the measurement station is clearly delayed. At $\mathrm{k}=0.2$, the appearance of the DSV occurs at close to the maximum $\alpha\left(\alpha=23^{\circ}\right)$ during the upstroke, while at $\mathrm{k}=0.6$ this occurs close to the minimum $\alpha\left(\alpha=7^{\circ}\right)$ in the downstroke. A further increase in $\mathrm{k}$ to 1.2 results in the DSV appearing back in the downstroke at $\alpha=18^{\circ}$. These data confirm the phase lag in the shedding of the DSV observed in the flow visualization pictures. The contours of figure 15 also indicate that the vortex induced velocities are the strongest around $\mathrm{k}=0.4$ which falls in the range of $\mathrm{k}$ where the dynamic stall phenomenon was inferred to be the strongest from the flow visualization photographs (section 3.1.2).

\section{Conclusions}

The following are the primary observations and inferences made in the present study.
(1) In addition to the well known dynamic stall vortex (DSV) forming on the suction surface, an intense vortex of opposite sense is observed to form near the trailing edge (TEV) just when the DSV is shed. The combined DSV and the TEV grow to a very large size and take the shape of a "mushroom" as they convect away from the airfoil. The transverse extent of this "mushroom" structure is the largest, more than two chords at two chord downstream of the trailing edge, for the intermediate $\mathrm{k}$ range of 0.2 to 0.4 . It has been known that the shedding of the DSV from the airfoil surface causes a sudden drop in lift which is followed by a rebounding of the lift in the lower branch of the hystersis loop of $\mathrm{C}_{\ell} \mathrm{vs}$. $\alpha$ curve. The present measurements and analysis show that this rebounding is caused by the passage of the TEV.

(2) The flowfield is documented in unprecedented detail using the phase averaging technique. The measured evolution of the vorticity field over a complete oscillation cycle agrees well with the flow visualization photographs.

(3) The unsteady circulation around the airfoil and therefore, the unsteady component of the lift, is estimated from the vorticity data by calculating the cumulative vorticity flux shed into the wake over one complete period of oscillation. The calculated unsteady lift is in reasonable agreement with the hystersis loop in the $\mathrm{C}_{\ell}$ versus $\alpha$ curve measured by McAlister et $\mathrm{al}^{27}$. The technique is a novel one, providing the unsteady aerodynamic forces strictly from wake measurements, and could be quite useful in future experiments of this kind.

(4) At very low reduced frequency $(k \leq 0.1)$, the oscillating airfoil behaves in a quasi-steady manner. Wake velocity spectra and flow visualization show the dominance of the bluff body shedding that would have occurred if the airfoil were held at the maximum angle of attack. As the oscillation frequency is increased, the bluff body shedding is gradually suppressed and the dynamic stall vortex and the trailing edge vortex become the primary features of the flowfield.

(5) For $\mathrm{k}=0.2$ the dynamic stall vortex is shed nearly at the maximum angle of attack of $25^{\circ}$, but as the reduced frequency is increased, the shedding of the DSV is progressively delayed. At $\mathrm{k}=0.8$ it is shed nearly at the minimum $\alpha$ of $5^{\circ}$, and at $\mathrm{k}=1.6$ two DSVs reside simultaneously on the suction surface during some part of the oscillation cycle.

\section{REFERENCE}

${ }^{1}$ McCroskey, W. J. 1982 Unsteady Airfoils. Annual Review of Fluid Mechanics, 14, 285-311.

${ }^{2}$ Carr, L. W. 1985 Dynamic Stall - Progress in Analysis and Prediction. Paper no. 85-1769-CP, AIAA Atmospheric Flight Mechanics Conference. 
${ }^{3}$ Carr, L. W., McAlister, K. W. and McCroskey, W. J. 1977 Analysis of the Development of Dynamic Stall Based on Oscillating Airfoil Experiments. NASA TN D-8382.

${ }^{4}$ Leishman, J.G. 1990 Dynamic Stall Experiments on the NACA 23012 Aerofoil. Experiments in Fluids, 9, 49-58.

${ }^{5}$ Mehta, U. B. 1978 Dynamic Stall of an Oscillating Airfoil. AGARD CP-227, Paper 23.

${ }^{6}$ Wu, J.-C., Kaza, K. R. V. and Sankar, L. N. 1987 A Technique for the Prediction of Airfoil Flutter Characteristics in Separated Flow. AIAA paper 87-0910.

${ }^{7}$ Koochesfahani, M. M. 1987 Vortical Patterns in the Wake of an Oscillating Airfoil. AIAA paper 87-0111.

${ }^{8} \mathrm{Ohmi}, \mathrm{k}$., coutanceau, M., Loc, T. P. and Dulieu, A. 1990 Vortex Formation Around an Oscillating and Translating Airfoil at Large Incidences. J. Fluid Mech. 211, 37-60.

${ }^{9} \mathrm{Gad}-\mathrm{el}-\mathrm{Hak}, \mathrm{M}$. and Ho, C.-M. 1986 Unsteady Vortical Flow Around Three-dimensional Lifting Surfaces. AIAA J. 24, no. 5, 713-721.

${ }^{10}$ Shida, Y., Kuwahara, K., Ono, K. and Takami, H. 1987 Computation of Dynamic Stall of a NACA0012 Airfoil. AIAA J. 25, 408-413.

${ }^{11}$ Visbal, M. R. and Shang, J. S. 1988 Investigation of the Flow Structure Around a Rapidly Pitching Airfoil. AIAA J. 27, no. 8.

${ }^{12}$ Reynolds, W. C. and Carr, L. W. 1985 Review of Unsteady, Driven, Separated Flows. AIAA paper no. 85-0527.

${ }^{13}$ Ho, C-M. 1986 An Alternative Look at the Unsteady Separation Phenomenon. Recent advances in aerodynamics, A. Krothappalli and C. A. Smith eds. Springer-Verlag, 165-178.

${ }^{14}$ Visbal, M. R. 1991 On the Formation and Control of the Dynamic Stall Vortex on a Pitching Airfoil. AIAA paper 91-0006.

${ }^{15}$ Methioulakis, D. S., Kim, M. J., Telionis, D. P. and Moor, D. T. 1985 On the Wake of a Pitching Airfoil. AIAA paper no. 85-1621.

${ }^{16}$ Booth, E. R. 1987 Measurement of Velocity and Vorticity Fields in the Wake of an Airfoil in Periodic Pitching Motion. NASA TP 2780.

${ }^{17}$ Cumpsty, N. A. 1989 Compressor Aerodynamics, Longman Scientific and Technical.

${ }^{18}$ Zaman, K. B. M. Q. and Hussain, A. K. M.

F. 1981 Taylor Hypothesis and Large-scale Coherent Structures. J. Fluid Mech. 112, 379-396.

${ }^{19}$ Panda, J. 1990 Experiments on Instabilities of Free Swirling and Non-swirling Free Jets. Ph. D. thesis, The Pennsylvania State University.

${ }^{20}$ Walker, J. M. and Robinson, M. C. 1990

Three-dimensional Driven Unsteady Separated Fflows.
IMACS 1st International Conference on Computational Physics, Univ. of Colorado, 236-239.

${ }^{21}$ Brendel, M. and Mueller, T.J., 1988 Boundary-layer Measurements on an Airfoil at Low Reynolds Numbers, J. Aircraft, 25, 612-617.

${ }^{22}$ Roshko, A. 1954 On the Drag and Shedding Frequency of Two-dimensional Bluff Bodies. NACA TN3169.

${ }^{23}$ McAlister, K.W., Carr, L.W. and McCroskey, W.J., 1978 Dynamic Stall Experiments on the NACA0012 Airfoil, NASA TP 1100.

${ }^{24}$ Kim, J. S. and Seung, O. P. 1988 Smoke Wire Visualization of Unsteady Separation Over an Oscillating Airfoil. ALAA J. 26, no. 41, 1408-1410.

${ }^{25}$ McAlister, K.W. and Carr, L.W. 1979 Water Tunnel Visualization of Dynamic Stall. J. of Fluids Eng. ASME transactions, 106, 376-380.

${ }^{26}$ Westphal, R.V. and Mehta, R.D. 1984 Crossed Hot-wire Data Acquisition and Reduction System. NASA TM 85871.

${ }^{27}$ McAlister, K.W., Pucci, S.L., McCroskey, W.L. and Carr, L.W., 1982 An Experimental Study of Dynamic Stall in Advanced Airfoil Sections, vol. 2, Pressure and Force Data, NASA TM 84245.

${ }^{28}$ Batchelor, G. K. 1967 An Introduction to Fluid Dynamics. Cambridge Univ. Press.

${ }^{29} \mathrm{Wu}$, J. C. 1981 Theory of Aerodynamic Force and Moments in Viscous Flows. ALAA J. 19, no. 14, 432-441.

${ }^{30}$ Wu, J.-C., Huff, D.L. and Sankar, L.N. 1990 Evaluation of Three Turbulence Models in Static Air Loads and Dynamic Stall Predictions. J. Aircraft, vol. 27, 382-384. 

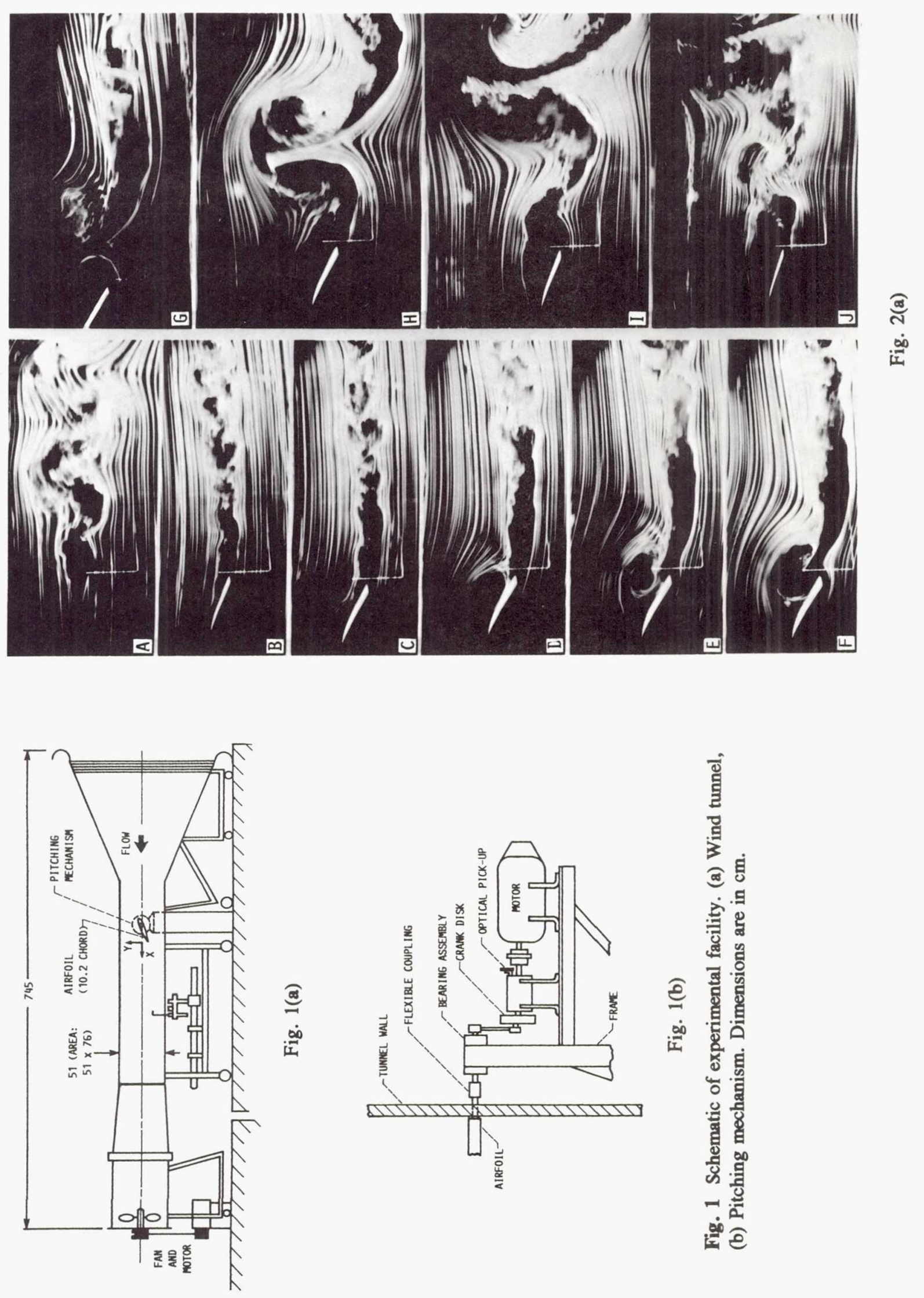


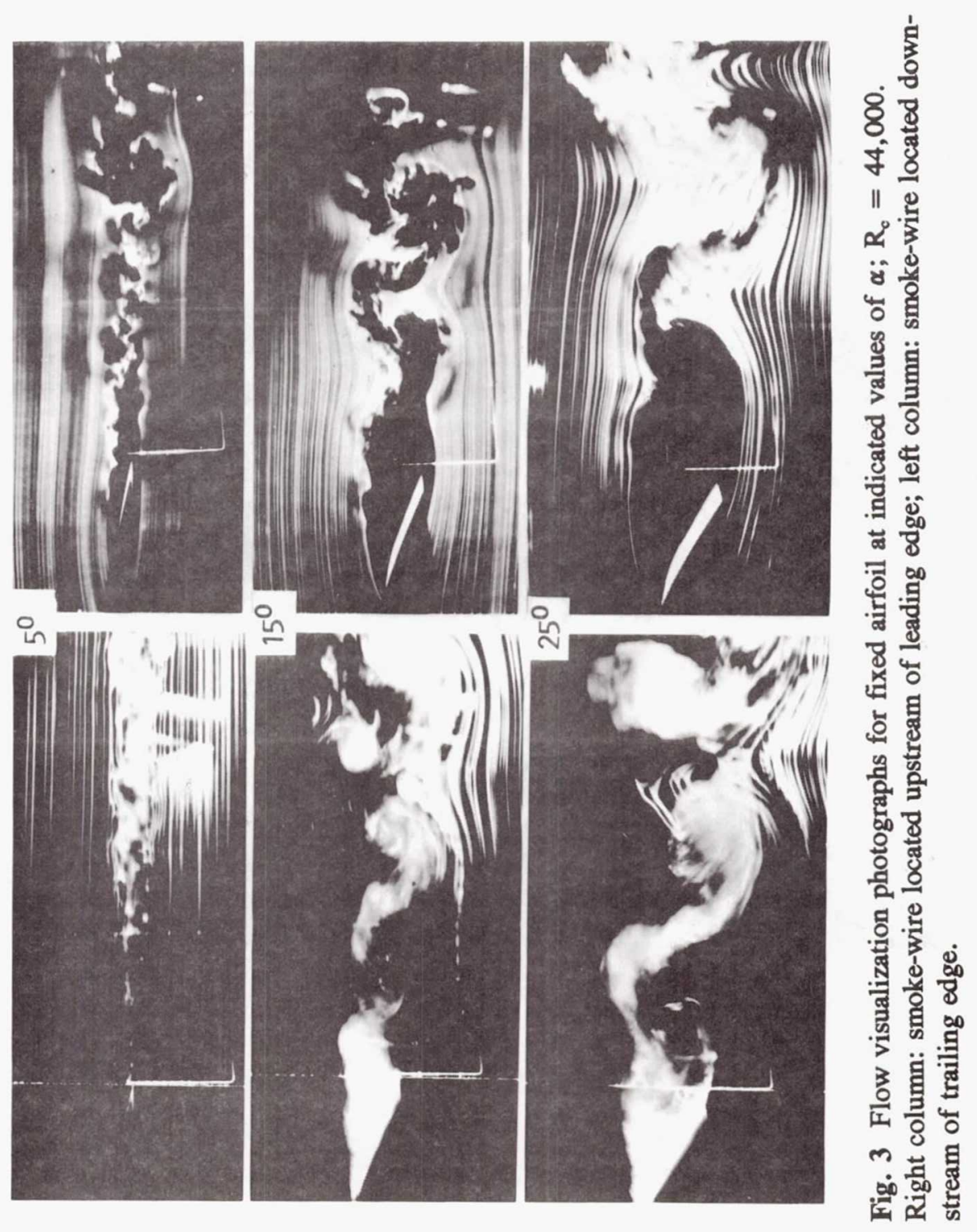

II

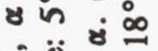

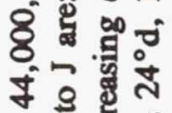

$11<\frac{8}{0} 0^{5}$

व

त हु

음.

II 氮密

$\checkmark$ of in

के घै

उ.

영 형 홍

坓豈:

务完

5 5 :

贯㝵

ช

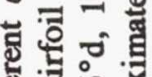

象的

可和

क

해용

要

을

동 농

들

정 त

零方

3 ส

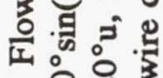

그으유

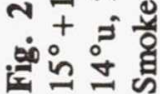

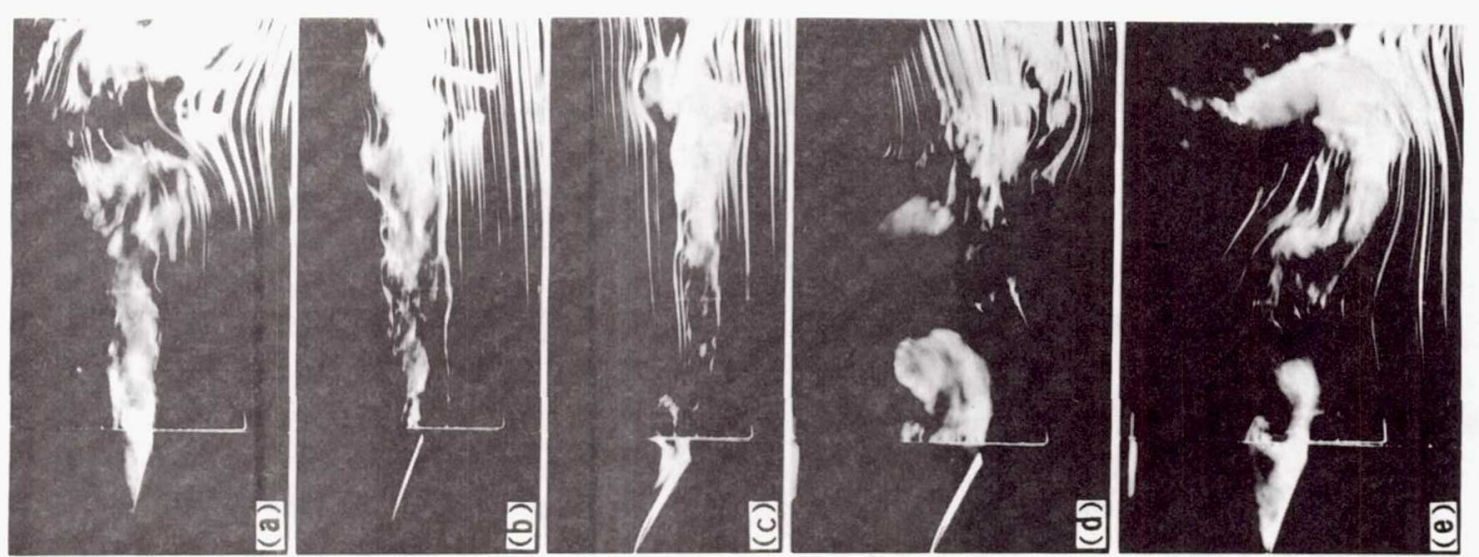

อู่ 

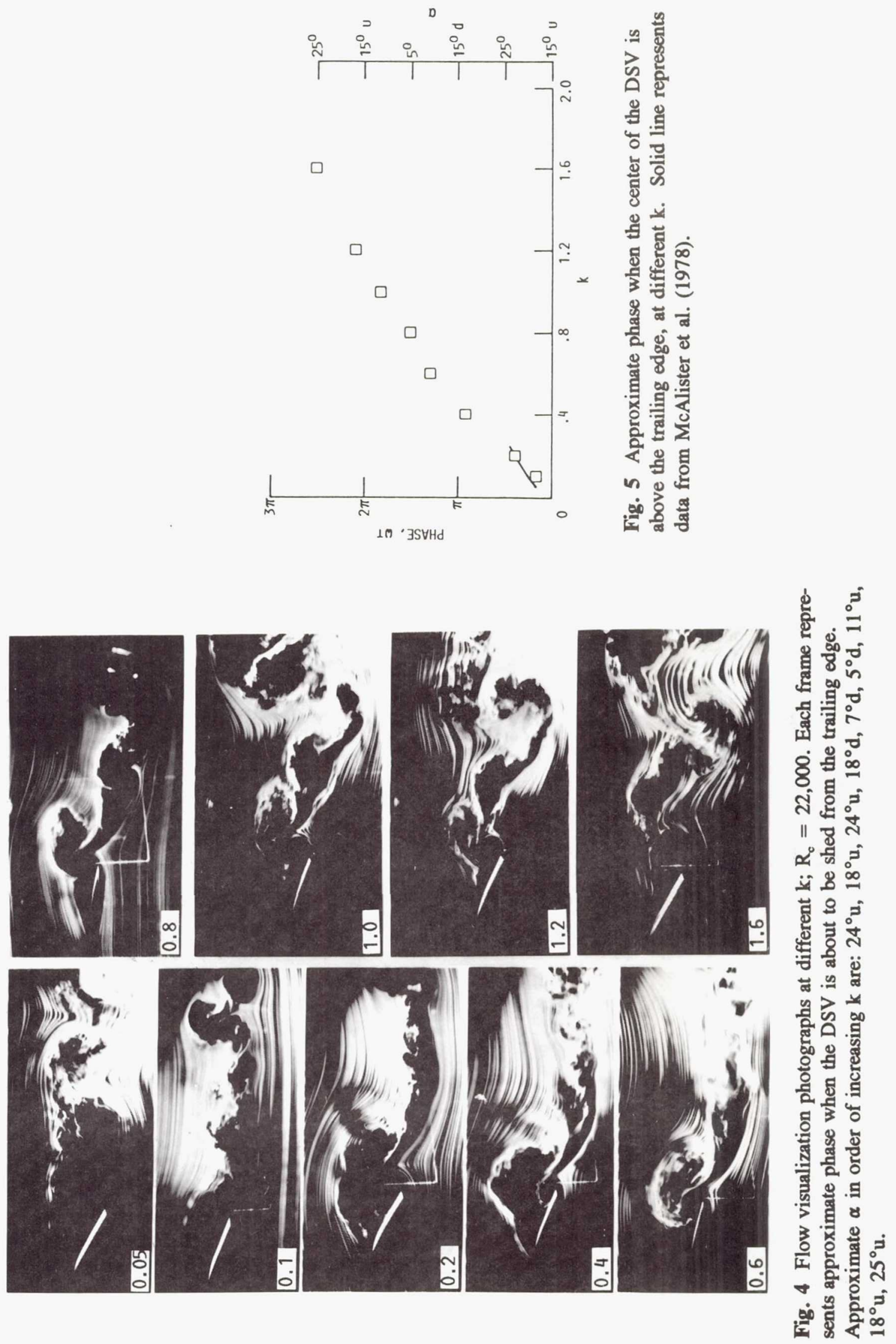

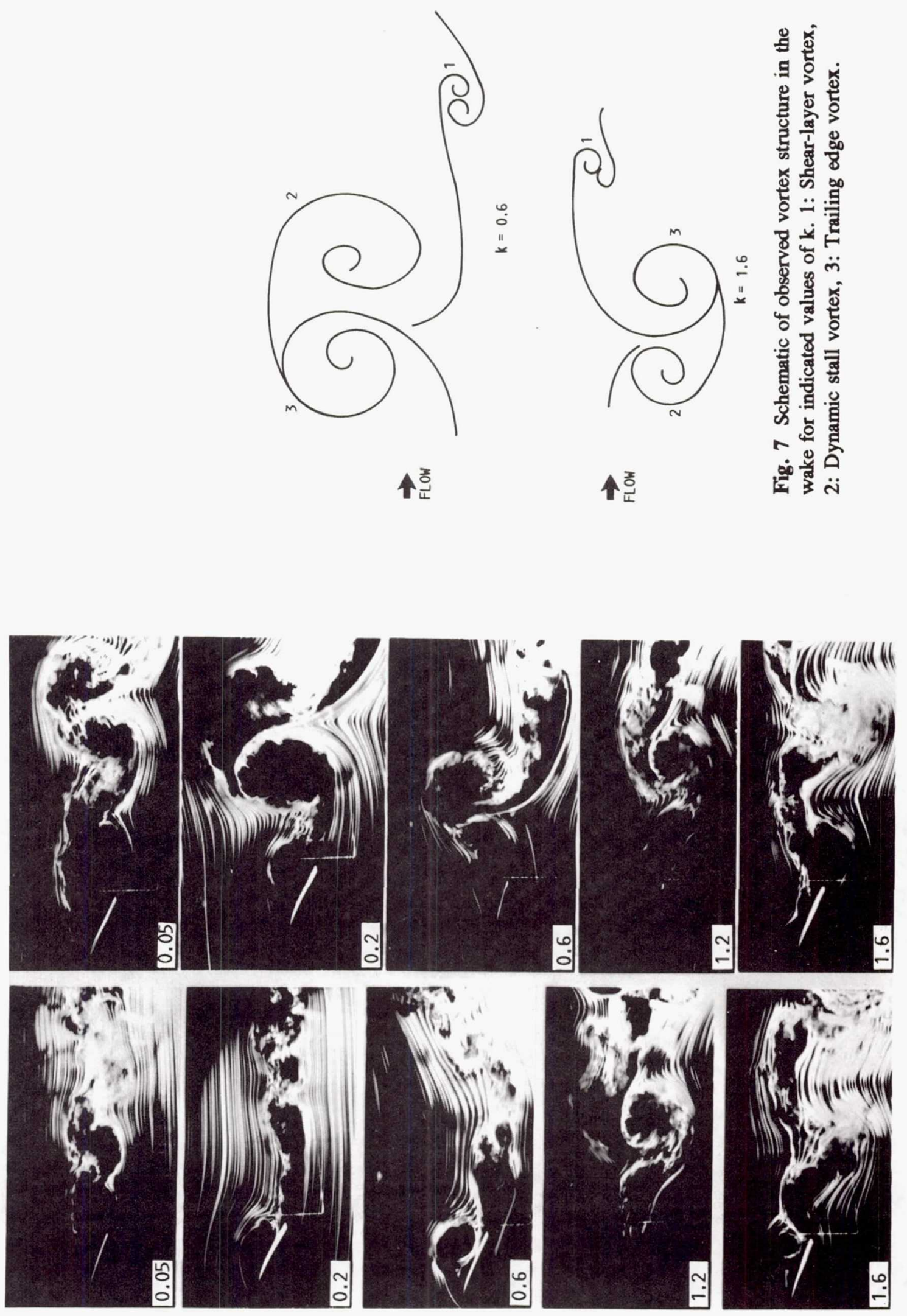

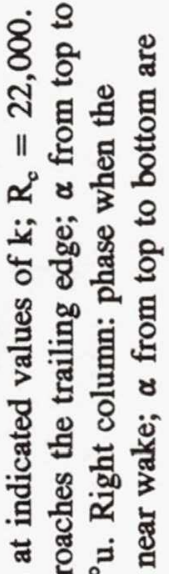

둘 을

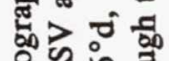

ํํ을

동

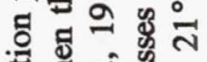
जี สี ส

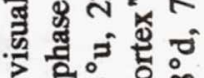
舟

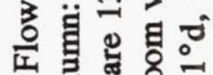
๑

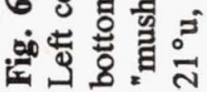




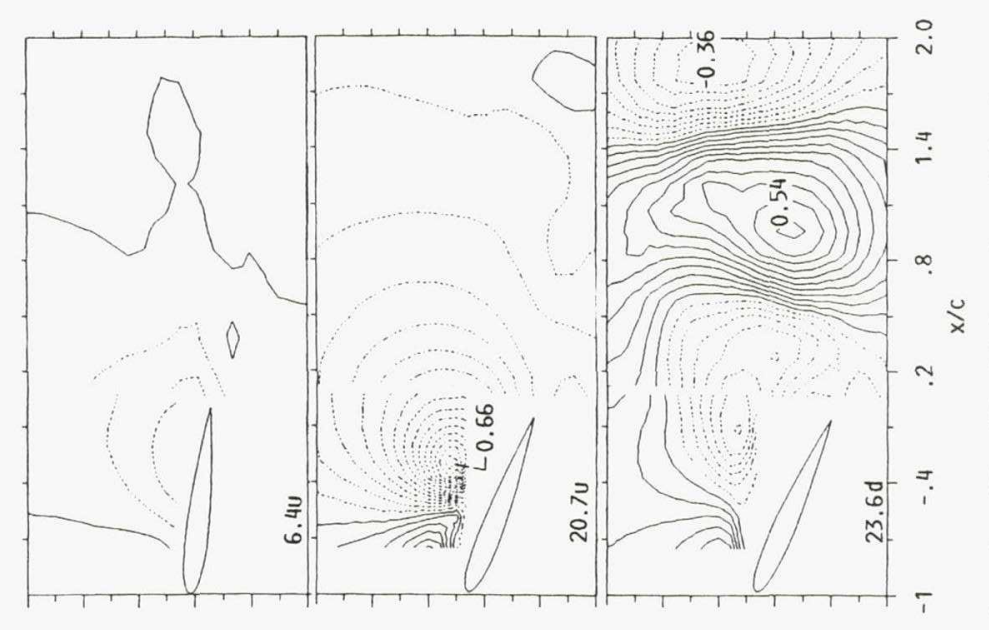

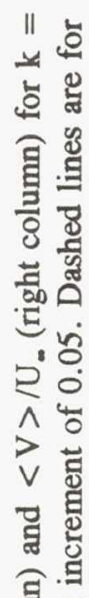
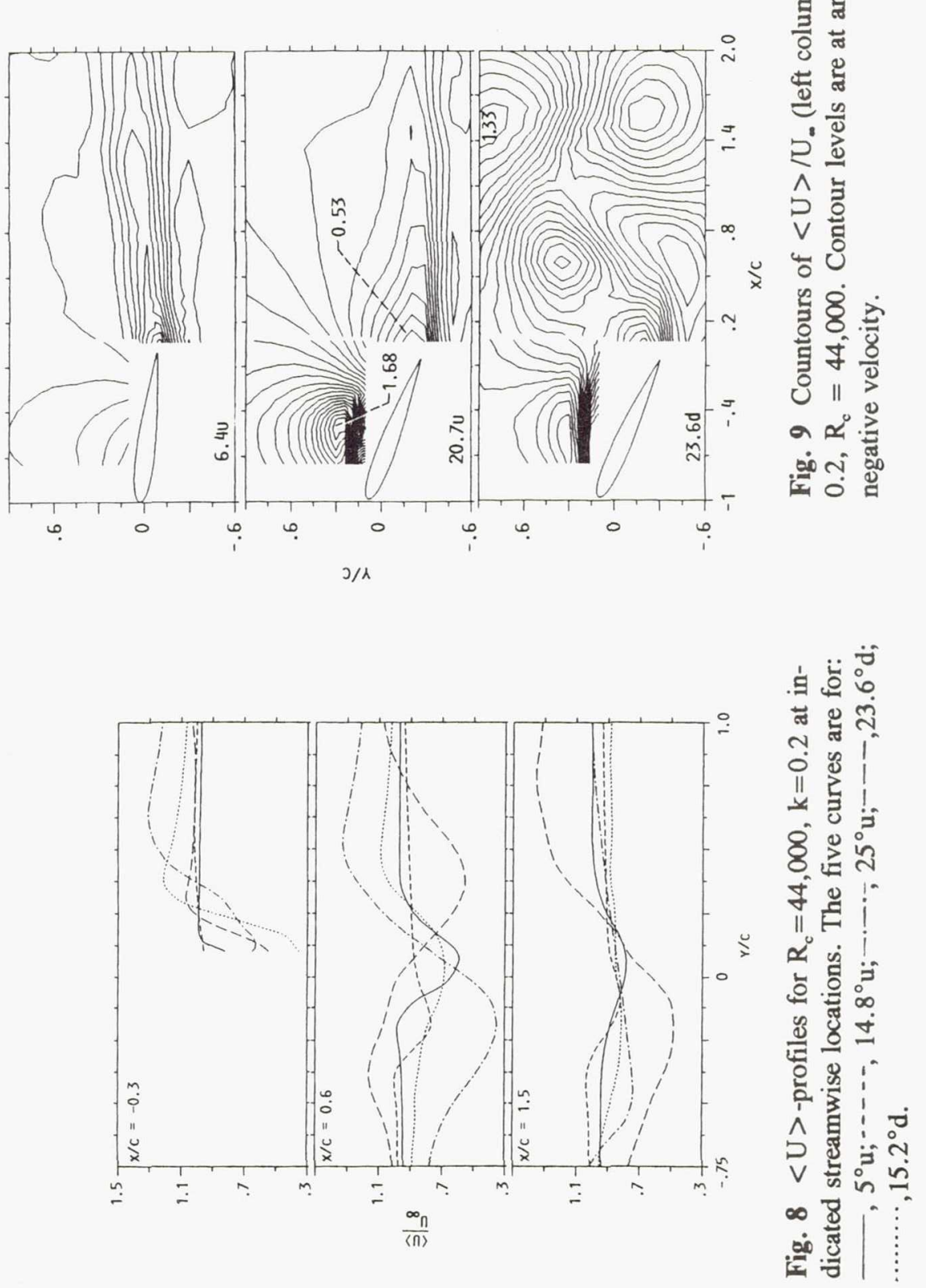


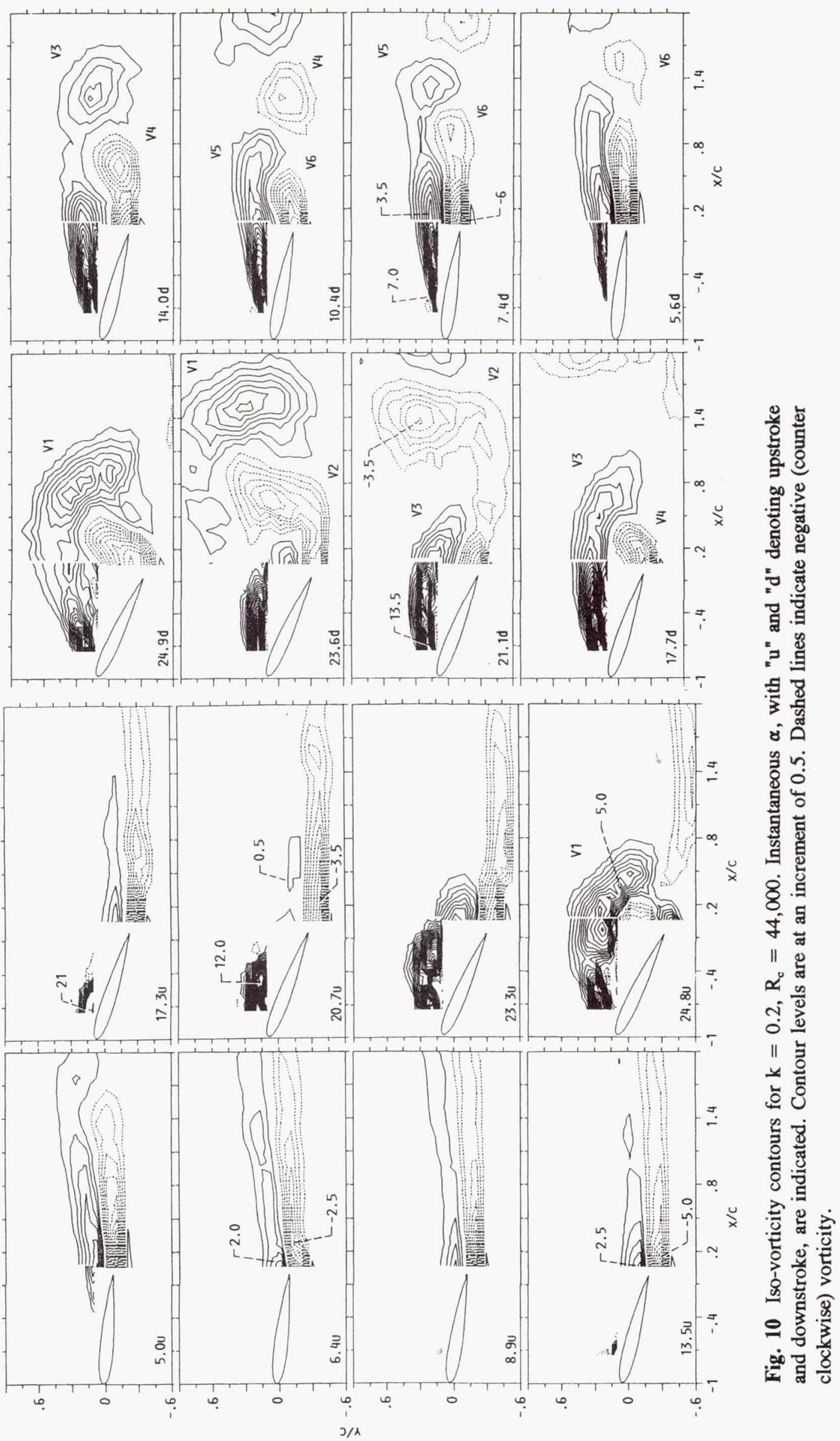



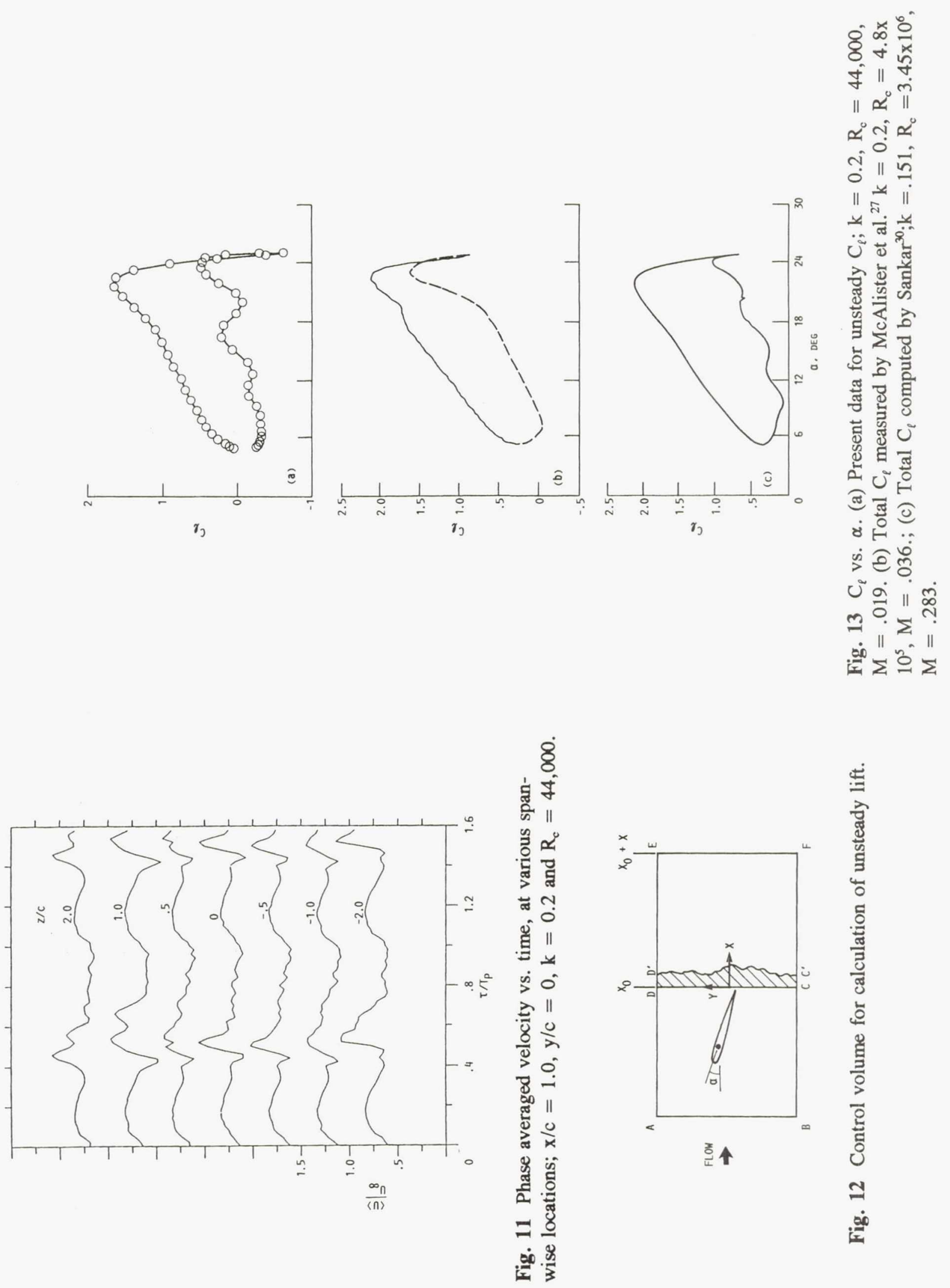

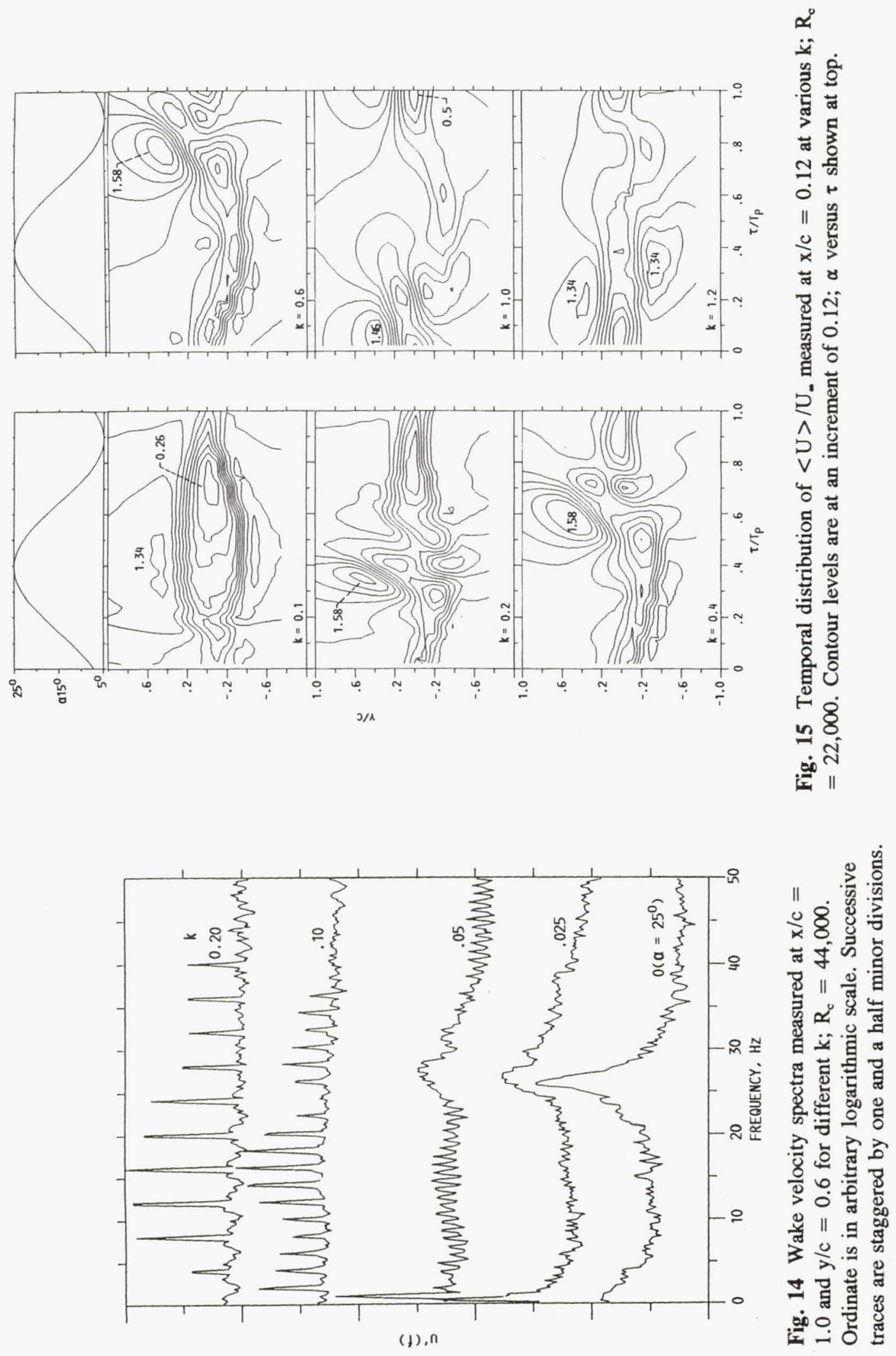
Public reporting burden for this collection of information is estimated to average 1 hour per response, including the time for reviewing instructions, searching existing data sources, gathering and maintaining the data needed, and completing and reviewing the collection of information. Send comments regarding this burden estimate or any other aspect of this collection of information, including suggestions for reducing this burden, to Washington Headquarters Services, Directorate for information Operations and Reports, 1215 Jefferson Davis Highway, Suite 1204, Arlington, VA 22202-4302, and to the Office of Management and Budget, Paperwork Reduction Project (0704-0188), Washington, DC 20503.

\begin{tabular}{|l|c|c|c|}
\hline 1. AGENCY USE ONLY (Leave blank) & $\begin{array}{c}\text { 2. REPORT DATE } \\
\text { June } 1992\end{array}$ & $\begin{array}{r}\text { 3. REPORT TYPE AND DATES COVERED } \\
\text { Technical Memorandum }\end{array}$ \\
\hline 4.
\end{tabular}

Experimental Investigation of the Flowfield of an Oscillating Airfoil 5. FUNDING NUMBERS

J. Panda and K.B.M.Q. Zaman

WU-505-62-52

7. PERFORMING ORGANIZATION NAME(S) AND ADDRESS(ES)

National Aeronautics and Space Administration

Lewis Research Center

Cleveland, Ohio 44135-3191

8. PERFORMING ORGANIZATION REPORT NUMBER

E-7046

9. SPONSORING/MONITORING AGENCY NAMES(S) AND ADDRESS(ES)

10. SPONSORING/MONITORING AGENCY REPORT NUMBER

National Aeronautics and Space Administration

Washington, D.C. 20546-0001

NASA TM-105675

\section{SUPPLEMENTARY NOTES}

Prepared for the 10th Applied Acrodynamics Conference, sponsored by American Institute of Acronautics and Astronautics, Palo Alto,

California, June 22-24, 1992. J. Panda, National Research Council-Resident Research Associate at NASA Lewis Research Center. K.B.M.Q

Zaman, NASA Lewis Research Center. Responsible person, J. Panda , (216) 433-8891.

12a. DISTRIBUTION/AVAILABILITY STATEMENT

12b. DISTRIBUTION CODE

Unclassified - Unlimited

Subject Category 02

\section{ABSTRACT (Maximum 200 words)}

The flowfield of an airfoil oscillated periodically over a wide range of reduced frequencies, $0 \leq \mathrm{k} \leq 1.6$ is studied experimentally at chord Reynolds numbers of $R_{c}=22,000$ and 44,000. The NACA0012 airfoil is pitched sinusoidally about one quarter chord between angles of attack $(\alpha)$ of $5^{\circ}$ and $25^{\circ}$. Detailed flow visualization and phase averaged vorticity measurements are carried out for $k=0.2$ to document the evolution and the shedding of the dynamic stall vortex (DSV). In addition to the DSV, an intense vortex of opposite sign originates from the trailing edge just when the DSV is shed. After being shed into the wake, the two together take the shape of a large "mushroom" while being convected away from the airfoil. The unsteady circulation around the airfoil and, therefore, the time varying component of the lift is estimated in a novel way from the shed vorticity flux and is found to be in good agreement with the lift variation reported by others. The delay in the shedding of the DSV with increasing $\mathrm{k}$, as observed by previous researchers, is documented for the full range of $k$. The DSV, for example, is shed nearly at the maximum $\alpha$ of $25^{\circ}$ at $k=0.2$, but is shed at the minimum $\alpha$ of $5^{\circ}$ at $k=0.8$. At low $k$, the flowfield appears quasisteady and the bluff body shedding corresponding to the maximum $\alpha\left(25^{\circ}\right)$ dominates the unsteady fluctuations in the wake.

\begin{tabular}{|c|c|}
\hline $\begin{array}{l}\text { 14. SUBJECT TERMS } \\
\text { Unsteady aerodynamics; Lift calculations }\end{array}$ \\
\hline $\begin{array}{c}\text { 17. SECURITY CLASSIFICATION } \\
\text { OF REPORT } \\
\text { Unclassified }\end{array}$ & $\begin{array}{c}\text { 18. SECURITY CLASSIFICATION } \\
\text { OF THIS PAGE } \\
\text { Unclassified }\end{array}$ \\
\hline
\end{tabular}

NSN 7540-01-280-5500

\begin{tabular}{|c|c|}
\hline & $\begin{array}{l}\text { 15. NUMBER OF PAGES } \\
20\end{array}$ \\
\hline & $\begin{array}{l}\text { 16. PRICE CODE } \\
\mathrm{AO} 3\end{array}$ \\
\hline $\begin{array}{l}\text { 19. SECURITY CLASSIFICATION } \\
\text { OF ABSTRACT } \\
\text { Unclassified }\end{array}$ & 20. LIMITATION OF ABSTRACT \\
\hline & $\begin{array}{l}\text { andard Form } 298 \text { (Rev. 2-89) } \\
\text { scribed by ANSI Std. Z39-18 } \\
3-102\end{array}$ \\
\hline
\end{tabular}

